\title{
The Relationship between Socially Prescribed Perfectionism and Academic Procrastination in Korea
}

\author{
$\bigcirc$ Choo Sang Yup • Oh Ji Eun • Lim Sung Moon \\ (Department of Psychology, Chungbuk National University, KOREA) \\ Key words: academic procrastination, perfectionism, achievement goals
}

Academic procrastination is defined as 'unnecessarily to put off starting and completing tasks in academic settings.' Procrastination is easily observed around us and associated with a variety of negative variables. Many researchers tried to find causes of procrastination, and then assumed that perfectionism might be a significant factor because they believed procrastination stems from excessively high standards. Especially, it was proposed by researchers that socially prescribed perfectionism might be a main cause of academic procrastination. However, the levels of the correlations between socially prescribed perfectionism and academic procrastination in Western studies were not so high, differently as expected. Additionally, the research in Korea reported much lower levels of correlations between them than the levels in Western studies or even no associations. These differences could be explained as the influences of cultural factors or/and any intervening variables in the relationship between socially prescribed perfectionism and academic procrastination.

On the basis of self-regulation, we explored possible variables to intervene in the relationship between the two variables. The self-regulation, as one of the theories to account for procrastination, assumes not that motivation directly lead to behavior, but that goals and strategies intervene in the relationship between motivation and behavior. In other words, self-regulation means that when individuals set up goals and approach the goals they monitor the process to ensure themselves to see whether they are adequately progressing toward their goals. As a result of our exploration for the possible variables in the light of self-regulation, achievement goals, cognitive strategies, and meta-cognitive strategies were expected to intervene between the two variables.

Thus, the purpose of this study was to examine the mediating effect of performance-avoidance goal in the relationship between socially prescribed perfectionism and academic procrastination. Furthermore, it was also investigated whether cognitive strategies and meta-cognitive strategies have any moderating effects in the process where performance-avoidance goal affects academic procrastination.

\section{METHOD}

Participants. 703 students in eight middle and high schools in Korea completed questionnaires. Males were 365(51.9\%) and females were $338(48.1 \%)$.

Measures. Academic procrastination was assessed with the 19-item Korean version of Aitken Procrastination Inventory (Aitken, 1982) whose Cronbach's alpha was .80. Socially prescribed perfectionism was assessed with the 45-item Korean version of Multidimensional Perfectionism Scale (Hewitt \& Flett, 1991) whose Cronbach's alpha was .71. Performance-avoidance goal was assessed with the 26-item Achievement Goal Orientation Scale (Park \& Lee, 2005) whose Cronbach's alpha was .82. Cognitive strategies and meta-cognitive strategies were assessed with the 27-item
Self-regulated Learning Scale (Yang, 2000) whose Cronbach's alphas were .84 and .75 respectively.

Statistical Analyses. First of all, the data were analyzed by Pearson Correlation Test to see associations among variables included. Next, for the analyses of the mediating and moderating effects, Baron and Kenny stepwise method and hierarchical regression were used.

\section{RESULTS and DISCUSSION}

As a result of correlation test, academic procrastination was positively related with socially prescribed perfectionism $(r$ $=.10, p<.01)$ and performance-avoidance goal $(r=.27$, $p<.001$ ), and negatively associated with cognitive strategies $(r=-.32, p<.001)$ and meta-cognitive strategies $(r=-.19$, $p<.001)$. Socially prescribed perfectionism was positively related with performance-avoidance goal $(r=.23, p<.001)$.

Performance-avoidance goal fully mediated the relationship between socially prescribed perfectionism and academic procrastination. This means that the levels of performance-avoidance goal increase as the levels of socially prescribed perfectionism rise and then the increased levels of performance-avoidance goal increase the levels of academic procrastination. Socially prescribed perfectionism and performance-avoidance goal accounted for about $7.3 \%$ of the variance in academic procrastination.

Cognitive strategies and meta-cognitive strategies moderated the relationship between performance-avoidance goal and academic procrastination. That is, the levels of academic procrastination became the lowest in the condition where the levels of performance-avoidance goal are low and the levels of cognitive strategies and meta-cognitive strategies are high.

Theses results suggest that counseling and educational interventions which can change students' performance-avoidance goal into mastery goal or performance-approach goal, and increase the levels of cognitive strategies and meta-cognitive strategies are necessary, in order to reduce the high levels of academic procrastination caused by socially prescribed perfectionism.

\section{REFERENCES}

Aitken, M. (1982). 'A personality profile of the college student procrastination.' Unpublished doctoral dissertation, University of Pittsburgh. PA, USA.

Hewitt, P. L. \& Flett, G. L. (1991). Perfectionism in the self and social contexts: Conceptualization, assessment, and association with psychopathology. Journal of Personality and Social Psychology, 60, 456-470.

Park, B. G. \& Lee, J. U. (2005). Development and validation of a $2 \times 2$ achievement goal orientation scale. The Korean Journal of Educational Psychology, 19, 327-352.

Yang, M. H. (2000). The study on the development and validation of self-regulated learning model. Unpublished doctoral dissertation. Seoul National University, Seoul, Korea. 